\title{
Optimal long-term investment in illiquid markets when prices have negative memory*
}

\author{
Lóránt Nagy ${ }^{\dagger} \quad$ Miklós Rásonyi ${ }^{\ddagger}$
}

\begin{abstract}
In a discrete-time financial market model with instantaneous price impact, we find an asymptotically optimal strategy for an investor maximizing her expected wealth. The asset price is assumed to follow a process with negative memory. We determine how the optimal growth rate depends on the impact parameter and on the covariance decay rate of the price.
\end{abstract}

Keywords: processes with negative memory; price impact; optimal investment; fractional Brownian motion.

MSC2020 subject classifications: 91G10; 91G80.

Submitted to ECP on June 30, 2020, final version accepted on March 17, 2021.

Supersedes arXiv: 2005.07080.

\section{Introduction}

Fractional Brownian motions (FBMs) with various Hurst parameters $H \in(0,1)$ have been enticing researchers of financial mathematics for a long time, since the appearance of [15] where such models for asset prices were suggested for the first time. Econometric literature eagerly investigated related models, see [2] for an early survey.

In idealistic models of trading, where market imperfections are disregarded, FBMs do not provide admissible models since they generate arbitrage opportunities (for $H \neq 1 / 2$ ), see [16], hence they quickly fell out of favour. Subsequent research revealed, however, that in the presence of market frictions, arbitrage disappears and FBMs become eligible candidates for describing prices, see [9] and [11].

Not only prices but also volatilites were also successfully modelled using FBMs, see e.g. [4, 7]. Note that, though market volatility is not an asset, trading so-called VIX futures is essentially equivalent to "trading" the volatility, see [14].

In markets with instantaneous price impact the first analysis of long-term investment for an asset price following an FBM has been carried out in [10]: the optimal growth rate of expected portfolio wealth has been found and an asymptotically optimal strategy has been exhibited. The robustness of such results was the next natural question: is the particular structure of FBMs needed for these conclusions? In [10] a larger class of Gaussian processes could also be treated where future increments are positively correlated to the past and the covariance structure is similar to that of FBMs with

\footnotetext{
*Supported by the "Lendület" grant LP 2015-6 of the Hungarian Academy of Sciences.

${ }^{\dagger}$ Central European University and Rényi Institute, Budapest, Hungary. E-mail: lorantnagy28@gmail . com

${ }^{\ddagger}$ Rényi Institute, Budapest, Hungary. E-mail: rasonyi@renyi. hu
} 
$H>1 / 2$. The question of extending the case of FBMs with $H<1 / 2$ to more general models remained open.

The current paper provides such an extension, based on more involved estimates than in the positively correlated case. For simplicity, we stay in a discrete-time setting. We derive conclusions similar to those of [10] in the case $H<1 / 2$, but this time for a larger class of Gaussian processes.

These models allow negative price values. As such, they can directly describe futures contracts. They can also be considered as stylized models reflecting important characteristics of a more general class of processes. Our theoretical results then give hints to approach more complex and realistic models as well. The situation is similar to that of the Bachelier model (allowing negative prices) and the Black-Scholes model: for many practical purposes they are equivalent, see e.g. [18].

Our results are asymptotic in the sense that conclusions are derived for long horizons, as in several standard settings of stochastic optimal control, see e.g. [12]. It should be noted that such long horizons indeed arise e.g. in high-frequency trading where investment strategies are executed at every millisecond and the trading interval is several hours long every day. In the more usual trading regime (with actions taken every day or every hour), the simulations of [10] show that our strategies perform as expected already on realistic time horizons (say, one year).

\section{Market model}

Let $(\Omega, \mathcal{F}, P)$ be a probability space equipped with a filtration $\mathcal{F}_{t}, t \in \mathbb{Z}$. Let $E[X]$ denote the expectation of a real-valued random variable $X$ (when exists).

We will consider a financial market where the price of a risky asset follows a process $S_{t}, t \in \mathbb{N}$, adapted to $\mathcal{F}_{t}, t \in \mathbb{N}$. The riskless asset is assumed to have price constant 1 .

Realistic modelling needs to take into account market frictions. We choose to be working in a market model with a temporary, nonlinear price impact where portfolios are penalized by the integral of a certain power of the trading speed. Such models were considered in $[1,3,6,13,17]$ in continuous time and in [5] in discrete time. These models subsume the popular choices of linear ( $\alpha=2$ in the discussion below) and square-root $(\alpha=3 / 2)$ price impact.

For some $T \in \mathbb{N}$ the class of feasible strategies up to terminal time $T$ is defined as

$$
\mathcal{S}(T):=\left\{\phi=\left(\phi_{t}\right)_{t=0}^{T}: \phi \text { is an } \mathbb{R} \text {-valued, adapted process }\right\} .
$$

As we will see, $\phi_{t}$ represents the change in the investor's position in the given asset (the "speed" of trading, to emphasize the analogy with continuous-time models). Let $z=\left(z^{0}, z^{1}\right) \in \mathbb{R}^{2}$ be a deterministic initial endowment where $z^{0}$ is in cash and $z^{1}$ is in the risky asset.

For a feasible strategy $\phi \in \mathcal{S}(T)$, the number of shares in the risky asset, with $\Phi_{0}=z^{1}$, at any time $t \geq 1$, is equal to

$$
\Phi_{t}:=z^{1}+\sum_{u=0}^{t-1} \phi_{u}
$$

For simplicity, we assume $z^{1}=0$ from now on, i.e. the initial number of shares is zero. We will shortly derive a similar formula for the cash position of the investor. In classical, frictionless models of trading, cash at time $T+1$ equals

$$
\sum_{u=1}^{T+1} \Phi_{u}\left(S_{u}-S_{u-1}\right)
$$


when starting from a 0 initial position. Algebraic manipulation of (2.3) yields

$$
\sum_{u=1}^{T+1} \Phi_{u}\left(S_{u}-S_{u-1}\right)=-\sum_{u=0}^{T} \phi_{u} S_{u}+S_{T+1} \sum_{u=0}^{T} \phi_{u} .
$$

We assume that price impact is a superlinear power function of the "trading speed" $\phi$ so we augment the above with a term that implements the effect of friction:

$$
-\sum_{u=0}^{T} \phi_{u} S_{u}+S_{T+1} \sum_{u=0}^{T} \phi_{u}-\sum_{u=0}^{T} \lambda\left|\phi_{u}\right|^{\alpha}
$$

where we assume $\alpha>1$ and $\lambda>0$. We wish to utilize only those portfolios where the risky asset is liquidated by the end of the trading period so we define

$$
\mathcal{G}(T):=\left\{\phi \in \mathcal{S}(T): \Phi_{T+1}=\sum_{u=0}^{T} \phi_{u}=0\right\} .
$$

Based on the previous discussion, for $\phi \in \mathcal{G}(T)$, the position in the riskless asset at time $T+1$ is defined by

$$
X_{T}(\phi):=z^{0}-\sum_{u=0}^{T} \phi_{u} S_{u}-\sum_{u=0}^{T} \lambda\left|\phi_{u}\right|^{\alpha} .
$$

For simplicity, we also assume $z^{0}=0$ from now on, i.e. portfolios start from nothing.

To investigate the potential of realizing monetary profits, we focus on a risk-neutral objective: a linear utility function. Let $x_{-}:=\max \{-x, 0\}$ for $x \in \mathbb{R}$. Define, for $T \in \mathbb{N}$,

$$
\mathcal{A}(T):=\left\{\phi \in \mathcal{G}(T): E\left[\left(X_{T}(\phi)\right)_{-}\right]<\infty\right\},
$$

the class of strategies starting from a zero initial position in both assets and ending at time $T+1$ in a cash only position with expected value greater than $-\infty$. The value of the problem we will consider is thus

$$
u(T):=\sup _{\phi \in \mathcal{A}(T)} E\left[X_{T}(\phi)\right] .
$$

The investors's objective is to find $\phi$ which, at least asymptotically as $T \rightarrow \infty$, achieves the same growth rate as $u(T)$.

\section{Asymptotically optimal investment}

First we introduce assumptions on the price process and its dependence structure.

Assumption 3.1. Let $Z_{t}, t \in \mathbb{Z}$ be a real-valued, zero-mean stationary Gaussian process which will represent price increments. Let $\mathcal{F}_{n}:=\sigma\left(Z_{i}, i \leq n\right)$ for $n \in \mathbb{N}$. Let $r(t):=$ $\operatorname{cov}\left(Z_{0}, Z_{t}\right), t \in \mathbb{Z}$ denote its covariance function. We assume that there exists $T_{0}>0$ and $J_{1}, J_{2}<0$ such that for all $t \geq T_{0}$,

$$
J_{1} t^{\chi} \leq r(t) \leq J_{2} t^{\chi}
$$

is satisfied for some parameter $\chi \in(-2,-1)$. Furthermore,

$$
\sum_{t \in \mathbb{Z}} r(t)=0 .
$$

Let us introduce the adapted price process defined by $S_{0}=0$ and $S_{t}=S_{t-1}+Z_{t}, t \geq 1$. 
Remark 3.2. Properties (3.1) and (3.2) express that $Z$ is a process with negative memory, see Definition 1.1.1 on page 1 of [8]. When $Z_{t}, t \in \mathbb{Z}$ are the increments of a FBM with Hurst parameter $H<1 / 2$, then (3.1) is satisfied with $\chi:=2 H-2$.

The next theorem is our main result: it provides the explicit form of an (asymptotically) optimal strategy and determines its expected asymptotic growth rate.

Theorem 3.3. Let Assumption 3.1 be in force. If $\lambda$ is small enough then maximal expected profits satisfy

$$
\limsup _{T \rightarrow \infty} \frac{u(T)}{T^{\left(\frac{\chi}{2}+1\right)\left(1+\frac{1}{\alpha-1}\right)+1}}<\infty
$$

and the strategy

$$
\phi_{t}(T, \alpha):=\left\{\begin{array}{cl}
-\operatorname{sgn}\left(S_{t}\right) \mid S_{t} t^{\frac{1}{\alpha-1}}, & 0 \leq t \leq 3\lfloor T / 6\rfloor, \\
-\frac{1}{3\lfloor T / 6\rfloor} \sum_{s=0}^{3\lfloor T / 6\rfloor} \phi_{s}, & 3\lfloor T / 6\rfloor<t \leq 6\lfloor T / 6\rfloor, \\
0, & \text { otherwise }
\end{array}\right.
$$

satisfies

$$
\liminf _{T \rightarrow \infty} \frac{E X_{T}(\phi(T, \alpha))}{T^{\left(\frac{\chi}{2}+1\right)\left(1+\frac{1}{\alpha-1}\right)+1}}>0 .
$$

Remark 3.4. The strategy above builds on the following intuition. In a market with friction one can not sell or buy with arbitrary speeds. Such behavior is punished in superlinear price-impact models, strategies that are not trading assets gradually can generate losses that ruin an otherwise profitable investment. Thus, liquidation must also be done at a careful pace. Our strategy operates as follows. On the first half of the given timeline it trades the underlying in a contrarian manner, that is going short when prices are high and entering long positions when low. It is intuitively clear that, due to the superlinear nature of friction, liquidation is best done with a constant speed. This is reflected in our strategy on the second half of the timeline.

\section{Proofs}

\subsection{General bounds for variance and covariance}

First we make some useful preliminary observations. Using stationarity of the increments of the process $S$, we have

$$
\begin{aligned}
\operatorname{var}\left(S_{t}\right) & =\operatorname{cov}\left(S_{t}, S_{t}\right)=\operatorname{cov}\left(\sum_{j=1}^{t} S_{j}-S_{j-1}, \sum_{i=1}^{t} S_{i}-S_{i-1}\right) \\
& =t \cdot \operatorname{var}\left(S_{1}-S_{0}\right)+2 \sum_{i=2}^{t} \sum_{j=1}^{i-1} \operatorname{cov}\left(S_{j}-S_{j-1}, S_{i}-S_{i-1}\right) \\
& =t \cdot \operatorname{var}\left(S_{1}-S_{0}\right)+2 \sum_{i=2}^{t} \sum_{j=1}^{i-1} \operatorname{cov}\left(S_{1}-S_{0}, S_{i-j+1}-S_{i-j}\right) \\
& =t \cdot r(0)+2 \sum_{i=2}^{t} \sum_{j=1}^{i-1} r(i-j) .
\end{aligned}
$$

Furthermore, for $s>t$ we similarly have

$$
\operatorname{cov}\left(S_{s}-S_{t}, S_{t}\right)=\sum_{i=t+1}^{s} \sum_{j=1}^{t} r(i-j) .
$$


Observe also that we can write

$$
r(0)=-2 \sum_{j=1}^{\infty} r(j)
$$

Turning to the variances, we first obtain a convenient expression for them. Note that for $i>1$ we have

$$
\sum_{j=1}^{i-1} r(i-j)=r(i-1)+\ldots+r(1)=r(1)+\ldots+r(i-1)=\sum_{j=1}^{i-1} r(j) .
$$

Using the observations (4.4), (4.1) and (4.3), we have

$$
\operatorname{var}\left(S_{t}\right)=-2 t \sum_{j=1}^{t-1} r(j)-2 t \sum_{j=t}^{\infty} r(j)+2 \sum_{i=2}^{t} \sum_{j=1}^{i-1} r(j),
$$

and algebraic manipulation of the summation operation $\left(-2 t \sum_{j=1}^{t-1}+2 \sum_{i=2}^{t} \sum_{j=1}^{i-1}\right)$ yields

$$
\begin{aligned}
& -2 t \sum_{j=1}^{t-1}+2 \sum_{i=2}^{t} \sum_{j=1}^{i-1} \\
& =-2 t\left(\sum_{j=1}^{T_{0}-1}+\sum_{j=T_{0}}^{t-1}\right)+2\left(\sum_{i=2}^{T_{0}-1}+\sum_{i=T_{0}}^{t}\right) \sum_{j=1}^{i-1} \\
& =-2 t \sum_{j=1}^{T_{0}-1}-2 t \sum_{j=T_{0}}^{t-1}+2 \sum_{i=2}^{T_{0}-1} \sum_{j=1}^{i-1}+2 \sum_{i=T_{0}}^{t} \sum_{j=1}^{i-1} \\
& =-2 t \sum_{j=1}^{T_{0}-1}-2 t \sum_{j=T_{0}}^{t-1}+2 \sum_{i=2}^{T_{0}-1} \sum_{j=1}^{i-1}+2 \sum_{j=1}^{T_{0}-1}+2 \sum_{i=T_{0}+1}^{t}\left(\sum_{j=1}^{T_{0}-1}+\sum_{j=T_{0}}^{i-1}\right) \\
& =-2 t \sum_{j=1}^{T_{0}-1}-2 t \sum_{j=T_{0}}^{t-1}+2 \sum_{i=2}^{T_{0}-1} \sum_{j=1}^{i-1}+2 \sum_{j=1}^{T_{0}-1}+2 \sum_{i=T_{0}+1}^{t} \sum_{j=1}^{T_{0}-1}+2 \sum_{i=T_{0}+1}^{t} \sum_{j=T_{0}}^{i-1} \\
& =-2 t \sum_{j=1}^{T_{0}-1}+2 \sum_{i=2}^{T_{0}-1} \sum_{j=1}^{i-1}+2 \sum_{j=1}^{T_{0}-1}+2 \sum_{i=T_{0}+1}^{t} \sum_{j=1}^{T_{0}-1}-2 t \sum_{j=T_{0}}^{t-1}+2 \sum_{i=T_{0}+1}^{t} \sum_{j=T_{0}}^{i-1},
\end{aligned}
$$

where the last line is only a reordering of terms. Setting $C_{1}=\sum_{j=1}^{T_{0}-1} r(j), C_{2}=$ $\sum_{i=2}^{T_{0}-1} \sum_{j=1}^{i-1} r(j)$ and $C_{3}=2\left(C_{2}-\left(T_{0}-1\right) C_{1}\right)$, the above calculation gives

$$
\begin{aligned}
\operatorname{var}\left(S_{t}\right) & =-2 t C_{1}+2 C_{2}+2 C_{1}+2\left(t-T_{0}\right) C_{1}+\left(-2 t \sum_{j=t}^{\infty}-2 t \sum_{j=T_{0}}^{t-1}+2 \sum_{i=T_{0}+1}^{t} \sum_{j=T_{0}}^{i-1}\right) r(j) \\
& =C_{3}+\left(-2 t \sum_{j=t}^{\infty}-2 t \sum_{j=T_{0}}^{t-1}+2 \sum_{i=T_{0}+1}^{t} \sum_{j=T_{0}}^{i-1}\right) r(j)
\end{aligned}
$$

From now on we will work with the parameter $H:=\frac{\chi}{2}+1$ for convenience. This parameter choice also refers back to the case of FBMs, see Remark 3.2.

Now we are ready to present three lemmas, providing a lower and an upper bound for the variance and an upper bound for the covariance. 
Lemma 4.1. There exist $T_{1} \in \mathbb{N}$ and $B_{1}>0$ such that for all $t \geq T_{1}$ we have

$$
\operatorname{var}\left(S_{t}\right) \geq B_{1} t^{2 H}
$$

Proof. Using properties induced by the choice of $T_{0}$ in Assumption 3.1 first note that

$$
\begin{aligned}
& \left(-2 t \sum_{j=T_{0}}^{t-1}+2 \sum_{i=T_{0}+1}^{t} \sum_{j=T_{0}}^{i-1}\right) r(j) \\
& \geq\left(-2 t \sum_{j=T_{0}}^{t-1}+2\left(t-T_{0}\right) \sum_{j=T_{0}}^{t-1}\right) r(j) \\
& =-2 T_{0} \sum_{j=T_{0}}^{t-1} r(j) \geq 0 .
\end{aligned}
$$

Also notice that

$$
\begin{aligned}
-2 t \sum_{j=t}^{\infty} r(j) & \geq-2 J_{2} t \sum_{j=t}^{\infty} j^{2 H-2} \geq-2 J_{2} t \int_{t}^{\infty} u^{2 H-2} d u \\
& =-2 J_{2} t \frac{1}{2 H-1}\left(-t^{2 H-1}\right)=\frac{2 J_{2}}{2 H-1} t^{2 H}
\end{aligned}
$$

Using these and (4.5)

$$
\operatorname{var}\left(S_{t}\right) \geq C_{3}+\frac{2 J_{2}}{2 H-1} t^{2 H}
$$

The threshold $T_{1}$ and the constant $B_{1}$ can be explicitly calculated in terms of the constants present in the above expression. This completes the proof.

Lemma 4.2. There exist $T_{2} \in \mathbb{N}$ and $B_{2}>0$ such that for all $t \geq T_{2}$ we have

$$
\operatorname{var}\left(S_{t}\right) \leq B_{2} t^{2 H}
$$

Proof. First note that algebraic manipulation of the operation $\left(-2 t \sum_{j=T_{0}}^{t-1}+2 \sum_{i=T_{0}+1}^{t} \times\right.$ $\left.\sum_{j=T_{0}}^{i-1}\right)$ yields

$$
\begin{aligned}
& -2 t \sum_{j=T_{0}}^{t-1}+2 \sum_{i=T_{0}+1}^{t} \sum_{j=T_{0}}^{i-1}=-2\left(t-T_{0}+T_{0}\right) \sum_{j=T_{0}}^{t-1}+2 \sum_{i=T_{0}}^{t-1} \sum_{j=T_{0}}^{i} \\
& =-2 \sum_{i=T_{0}}^{t-1} \sum_{j=T_{0}}^{t-1}+2 \sum_{i=T_{0}}^{t-1} \sum_{j=T_{0}}^{i}-2 T_{0} \sum_{j=T_{0}}^{t-1}=-2 \sum_{i=T_{0}}^{t-1}\left(\sum_{j=T_{0}}^{t-1}-\sum_{j=T_{0}}^{i}\right)-2 T_{0} \sum_{j=T_{0}}^{t-1} \\
& =-2 \sum_{i=T_{0}}^{t-1} \sum_{j=i+1}^{t-1}-2 T_{0} \sum_{j=T_{0}}^{t-1} .
\end{aligned}
$$


By Assumption 3.1, this implies

$$
\begin{aligned}
& \left(-2 t \sum_{j=T_{0}}^{t-1}+2 \sum_{i=T_{0}+1}^{t} \sum_{j=T_{0}}^{i-1}\right) r(j) \leq-2 J_{1}\left(\sum_{i=T_{0}}^{t-1} \sum_{j=i+1}^{t-1} j^{2 H-2}+T_{0} \sum_{j=T_{0}}^{t-1} j^{2 H-2}\right) \\
& \leq-2 J_{1}\left(\sum_{i=T_{0}}^{t-1} \int_{i}^{t-1} u^{2 H-2} d u+T_{0} \int_{T_{0}-1}^{t-1} u^{2 H-2} d u\right) \\
& =-\frac{2 J_{1}}{2 H-1}\left(\sum_{i=T_{0}}^{t-1}\left((t-1)^{2 H-1}-i^{2 H-1}\right)+T_{0}\left((t-1)^{2 H-1}-\left(T_{0}-1\right)^{2 H-1}\right)\right) \\
& =-\frac{2 J_{1}}{2 H-1}\left(t(t-1)^{2 H-1}-\sum_{i=T_{0}}^{t-1} i^{2 H-1}-T_{0}\left(T_{0}-1\right)^{2 H-1}\right) \\
& \leq \frac{2 J_{1}}{2 H-1} \sum_{i=T_{0}}^{t-1} i^{2 H-1}+\frac{2 J_{1}}{2 H-1} T_{0}\left(T_{0}-1\right)^{2 H-1} \\
& \leq \frac{2 J_{1}}{2 H(2 H-1)}\left((t-1)^{2 H}-\left(T_{0}-1\right)^{2 H}\right)+\frac{2 J_{1}}{2 H-1} T_{0}\left(T_{0}-1\right)^{2 H-1} \\
& \leq \frac{2 J_{1}}{2 H(2 H-1)} t^{2 H}+\frac{2 J_{1}}{2 H-1} T_{0}\left(T_{0}-1\right)^{2 H-1} \cdot
\end{aligned}
$$

To proceed observe that, using the asymptotics in Assumption 3.1, for $t>2$ we have

$$
\begin{aligned}
-2 t \sum_{j=t}^{\infty} r & (j) \leq-2 J_{1} t \sum_{j=t}^{\infty} j^{2 H-2} \leq-2 J_{1} t \int_{t-1}^{\infty} u^{2 H-2} d u \\
& =\frac{2 J_{1} t}{2 H-1}(t-1)^{2 H-1} \leq \frac{2 J_{1} t}{2 H-1}(t-t / 2)^{2 H-1} \\
& =\frac{2^{2-2 H} J_{1}}{2 H-1} t^{2 H} .
\end{aligned}
$$

These results yield for $t>\max \left(2, T_{0}\right)$, using again (4.5), that

$$
\operatorname{var}\left(S_{t}\right) \leq C_{3}+\left(\frac{2 J_{1}}{2 H(2 H-1)}+\frac{2^{2-2 H} J_{1}}{2 H-1}\right) t^{2 H}+\frac{2 J_{1}}{2 H-1} T_{0}\left(T_{0}-1\right)^{2 H-1}
$$

The threshold $T_{2}$ and the constant $B_{2}$ could again be explicitly given. The proof is complete.

We proceed with the lemma controlling the covariance $\operatorname{cov}\left(S_{s}-S_{t}, S_{t}\right)$.

Lemma 4.3. There exist $T_{3} \in \mathbb{N}$ and $D_{1}, D_{2}>0$ such that

$$
\operatorname{cov}\left(S_{s}-S_{t}, S_{t}\right) \leq D_{1} \text { for all } s>t>T_{3}
$$

For a fixed $v>1$, define

$$
U(v):=J_{2}(2 H)^{-1}(2 H-1)^{-1}\left(1-\left(v^{2 H}-(v-1)^{2 H}\right)\right) .
$$

Then

$$
\operatorname{cov}\left(S_{s}-S_{t}, S_{t}\right) \leq D_{2}-U(v) t^{2 H}<0 \text { holds for all } s>t>T_{3} \text { satisfying } \frac{s}{t}>v .
$$

There exists $K>1$ and $T_{4} \in \mathbb{N}$ such that

$$
\operatorname{cov}\left(S_{s}-S_{t}, S_{t}\right) \leq 0 \text { for all } s>t>T_{4} \text { satisfying } s-t>K .
$$


Proof. Let us set

$$
C_{4}=\sum_{j=-T_{0}+1}^{0} \sum_{i=1}^{1+T_{0}} r(i-j), \quad C_{5}=J_{2} \sum_{j=-T_{0}+1}^{0} \sum_{i=1}^{1+T_{0}}(i-j)^{2 H-2},
$$

and define $C_{6}=C_{4}-C_{5}$. Note that, for each $t \in \mathbb{N}, C_{4}=\sum_{j=t-T_{0}+1}^{t} \sum_{i=t+1}^{t+1+T_{0}} r(i-j)$, and $C_{5}=J_{2} \sum_{j=t-T_{0}+1}^{t} \sum_{i=t+1}^{t+1+T_{0}}(i-j)^{2 H-2}$. For $t>T_{0}$, we have

$$
\begin{aligned}
\operatorname{cov}\left(S_{s}\right. & \left.-S_{t}, S_{t}\right)=\sum_{j=1}^{t} \sum_{i=t+1}^{s} r(i-j) \\
& \leq C_{6}+J_{2} \sum_{j=1}^{t} \sum_{i=t+1}^{s}(i-j)^{2 H-2} \leq C_{6}+J_{2} \sum_{j=1}^{t} \int_{t+1-j}^{s+1-j} u^{2 H-2} d u \\
& \leq C_{6}+\frac{J_{2}}{2 H-1} \sum_{j=1}^{t}\left((s+1-j)^{2 H-1}-(t+1-j)^{2 H-1}\right) \\
& =C_{6}+\frac{J_{2}}{2 H-1} \sum_{j=1}^{t}(s+1-j)^{2 H-1}-\frac{J_{2}}{2 H-1} \sum_{j=1}^{t}(t+1-j)^{2 H-1} \\
& \leq C_{6}+\frac{J_{2}}{2 H-1} \int_{s-t}^{s} u^{2 H-1} d u-\frac{J_{2}}{2 H-1} \int_{1}^{t+1} u^{2 H-1} d u \\
& =C_{6}+\frac{J_{2}}{2 H(2 H-1)}\left(s^{2 H}-(s-t)^{2 H}\right)-\frac{J_{2}}{2 H(2 H-1)}\left((t+1)^{2 H}-1\right) \\
& =C_{6}+\frac{J_{2}}{2 H(2 H-1)}\left(s^{2 H}-(s-t)^{2 H}-\left((t+1)^{2 H}-1\right)\right) . \\
& =: C_{6}+C_{7}\left(s^{2 H}-(s-t)^{2 H}-\left((t+1)^{2 H}-1\right)\right) .
\end{aligned}
$$

Since $s \geq t+1$ the expression $C_{7}\left(s^{2 H}-(s-t)^{2 H}-\left((t+1)^{2 H}-1\right)\right)$ is non-positive, which yields

$$
\operatorname{cov}\left(S_{s}-S_{t}, S_{t}\right) \leq C_{6},
$$

proving the first statement of the lemma. Now, for all $v>1$ the property $\frac{s}{t}>v$-together with the previous constraint of $t>T_{0}$ - further implies

$$
\begin{aligned}
\operatorname{cov}\left(S_{s}\right. & \left.-S_{t}, S_{t}\right) \leq C_{6}+C_{7}\left(s^{2 H}-(s-t)^{2 H}-\left((t+1)^{2 H}-1\right)\right) \\
& \leq C_{6}+C_{7}\left(\left(v^{2 H}-(v-1)^{2 H}-1\right) t^{2 H}+1\right) \\
& =C_{6}+C_{7}+C_{7}\left(v^{2 H}-(v-1)^{2 H}-1\right) t^{2 H} .
\end{aligned}
$$

Obviously, for large enough $t$ the bound becomes strictly negative, proving the second statement. Now, assuming $s-t \geq K>1$ beside $t>T_{0}$ we have

$$
\begin{aligned}
\operatorname{cov}\left(S_{s}\right. & \left.-S_{t}, S_{t}\right) \leq C_{6}+C_{7}\left((t+K)^{2 H}-K^{2 H}-\left((t+1)^{2 H}-1\right)\right) \\
& =C_{6}-C_{7}\left(K^{2 H}-1\right)+C_{7}\left((t+K)^{2 H}-(t+1)^{2 H}\right) \\
& \leq C_{6}-C_{7}\left(K^{2 H}-1\right)+C_{7} 2 H K t^{2 H-1} .
\end{aligned}
$$

This shows that $K$ can be chosen so large that $C_{6}-C_{7}\left(K^{2 H}-1\right)<0$ and then, since $2 H-1<0$, a threshold $T_{4}$ - depending on $K$ - for the variable $t$ can be specified so that

$$
C_{6}-C_{7}\left(K^{2 H}-1\right)+C_{7} 2 H K t^{2 H-1} \leq 0
$$

whenever $t$ exceeds the threshold, proving the third statement, completing the proof of the lemma. 


\subsection{Key estimates}

Define

$$
\rho(s, t):=\frac{\operatorname{cov}\left(S_{s}, S_{t}\right)}{\operatorname{var}\left(S_{t}\right)}=\frac{\operatorname{cov}\left(S_{s}-S_{t}, S_{t}\right)}{\operatorname{var}\left(S_{t}\right)}+1, s \in \mathbb{N}, t \in \mathbb{N} \backslash\{0\} .
$$

Lemma 4.4. There exist $\bar{T} \in \mathbb{N}$ and constants $R>0, K>1, \eta \in(1 / 2,1)$ and $\varepsilon>0$ such that

1. $\rho(s, t)<1+R$, for all $t<s$;

2. $\rho(s, t) \leq 1$, whenever $\bar{T}<t<s$ and $s-t>K$;

3. For all $T \in \mathbb{N}, \rho(s, t) \leq 1-\varepsilon$, whenever $\bar{T}<t<\frac{T}{2}<\eta T<s$. Furthermore, one can also guarantee $T / 2+K<\eta T$ in this case.

Proof of Lemma 4.4. Let $B_{2}, U(\cdot), T_{1}, T_{2}, T_{3}, T_{4}, D_{1}, D_{2}$ and $K$ be as in Lemma 4.2 and Lemma 4.3. Choose $T^{\prime}>\max \left\{T_{1}, T_{2}, T_{3}\right\}$ so large that $\frac{D_{2}}{B_{2}}\left(T^{\prime}\right)^{-2 H}-\frac{U(4 / 3)}{B_{2}}<0$ and set $\eta:=2 / 3$. Lemma 4.2 and Lemma 4.3 now show that whenever $T^{\prime}<t<T / 2$ and $s \in(\eta T, T)$, we have

$$
\frac{\operatorname{cov}\left(S_{s}-S_{t}, S_{t}\right)}{\operatorname{var}\left(S_{t}\right)} \leq \frac{D_{2}}{B_{2}} t^{-2 H}-\frac{U(4 / 3)}{B_{2}} \leq \frac{D_{2}}{B_{2}}\left(T^{\prime}\right)^{-2 H}-\frac{U(4 / 3)}{B_{2}},
$$

which yields $\rho(s, t) \leq 1-\varepsilon$, where $\varepsilon=-\frac{D_{2}}{B_{2}}\left(T^{\prime}\right)^{-2 H}+\frac{U(4 / 3)}{B_{2}}$. Lemma 4.3 shows that $t>T_{4}$, ensures that $s-t>K$ implies $\rho(s, t) \leq 1$. Finally, set $\bar{T}=\max \left\{T^{\prime}, T_{4}, 3 K\right\}$. It is clear - using (4.7) in the proof of Lemma 4.3 - that for fixed $t$, the function $(s, t) \mapsto \rho(s, t)$ is bounded. So let $D_{1}^{\prime}=\max _{0<t<\bar{T}} \sup _{s>0} \rho(s, t)$ and define $R=\max \left\{D_{1}, D_{1}^{\prime}\right\}-1$ It remains to guarantee $T / 2+K<\eta T$ but this follows since $\bar{T}<t<T / 2$ implies $T>6 K$. The quantities $\eta, \bar{T}, R, K$ and $\varepsilon$ constructed above fulfill all the requirements.

Proof of Theorem 3.3. First we determine the maximal expected growth rate of portfolios. Let us define

$$
Q(T)=\sum_{t=0}^{T} E\left|S_{t}\right|^{\frac{\alpha}{\alpha-1}}
$$

Let $G(x):=\lambda|x|^{\alpha}, x \in \mathbb{R}$ and denote its Fenchel-Legendre conjugate

$$
G^{*}(y):=\sup _{x \in \mathbb{R}}(x y-G(x))=\frac{\alpha-1}{\alpha} \alpha^{\frac{1}{1-\alpha}} \lambda^{\frac{1}{1-\alpha}}|y|^{\frac{\alpha}{\alpha-1}}, \quad y \in \mathbb{R} .
$$

By definition of $G^{*}$, for all $\phi \in \mathcal{G}(T)$,

$$
X_{T}(\phi) \leq \sum_{t=0}^{T} G^{*}\left(-S_{t}\right)=C \sum_{t=0}^{T}\left|S_{t}\right|^{\alpha /(\alpha-1)}
$$

for some $C>0$ and hence

$$
E X_{T}(\phi) \leq C Q(T)<\infty .
$$

Note that this bound is independent of $\phi$. Using Lemma 4.2 it holds that

$$
\begin{aligned}
Q(T) & =C_{\frac{\alpha}{\alpha-1}} \sum_{t=0}^{T} \operatorname{var}\left(S_{t}\right)^{\frac{\alpha}{2(\alpha-1)}} \\
& \leq C_{\frac{\alpha}{\alpha-1}} \sum_{t=0}^{T_{2}-1} \operatorname{var}\left(S_{t}\right)^{\frac{\alpha}{2(\alpha-1)}}+C_{\frac{\alpha}{\alpha-1}} B_{2} \sum_{t=T_{2}}^{T} t^{\frac{H \alpha}{(\alpha-1)}} \\
& \leq C_{\frac{\alpha}{\alpha-1}, T_{2}}+C_{\alpha, H, B_{2}} T^{H\left(1+\frac{1}{\alpha-1}\right)+1}
\end{aligned}
$$


Thus the maximal expected profit grows as $T^{H\left(1+\frac{1}{\alpha-1}\right)+1}$ with the power of the horizon, this proves (3.3). Now, untill further notice, let $T$ be a multiple of 6 . With the strategy defined in (3.4), the dynamics takes the form

$$
\begin{aligned}
X_{T}(\phi)= & \sum_{t=0}^{T / 2}\left|S_{t}\right|^{\frac{\alpha}{\alpha-1}} \\
& -\sum_{t=0}^{T / 2} \lambda\left|S_{t}\right|^{\frac{\alpha}{\alpha-1}} \\
& -\frac{1}{T / 2} \sum_{s=T / 2+1}^{T} S_{s} \sum_{t=0}^{T / 2} \operatorname{sgn}\left(S_{t}\right)\left|S_{t}\right|^{\frac{1}{\alpha-1}} \\
& -\left.\left.\frac{1}{T / 2} \sum_{s=T / 2+1}^{T} \lambda\left|\sum_{t=0}^{T / 2} \operatorname{sgn}\left(S_{t}\right)\right| S_{t}\right|^{\frac{1}{\alpha-1}}\right|^{\alpha} .
\end{aligned}
$$

In the above expression let us denote the four terms by $I_{1}(T), I_{2}(T), I_{3}(T), I_{4}(T)$, respectively, so that

$$
X_{T}(\phi)=I_{1}(T)-I_{2}(T)-I_{3}(T)-I_{4}(T) .
$$

The upper bound constructed in (4.13) for $Q(T)$ right away gives us an upper estimate for $E I_{1}(T)$ as $E I_{1}(T)=Q(T / 2)$. Using Lemma 4.1, we likewise present a lower estimate as

$$
\begin{aligned}
Q(T / 2)=E\left[I_{1}(T)\right] & =C_{\frac{\alpha}{\alpha-1}} \sum_{t=0}^{T / 2} \operatorname{var}\left(S_{t}\right)^{\frac{\alpha}{2(\alpha-1)}} \\
& \geq C_{\frac{\alpha}{\alpha-1}} \sum_{t=0}^{T_{1}-1} \operatorname{var}\left(S_{t}\right)^{\frac{\alpha}{2(\alpha-1)}}+C_{\frac{\alpha}{\alpha-1}} B_{1} \sum_{t=T_{1}}^{T / 2} t^{\frac{H \alpha}{\alpha-1}} \\
& \geq C_{\frac{\alpha}{\alpha-1}, H, B_{1}, T_{1}}+C_{\frac{\alpha}{\alpha-1}, H, B_{1}} T^{H\left(1+\frac{1}{\alpha-1}\right)+1},
\end{aligned}
$$

To treat the terms $I_{2}(T)$ and $I_{4}(T)$, note that with $\alpha>1$ the function $x \mapsto|x|^{\alpha}$ is convex, thus applying Jensen's inequality

$$
\left|E I_{4}(T)\right| \leq E\left|I_{2}(T)\right|=\lambda E\left[\sum_{t=0}^{T / 2}\left|S_{t}\right|^{\frac{\alpha}{\alpha-1}}\right]=\lambda \sum_{t=0}^{T / 2} E\left|S_{t}\right|^{\frac{\alpha}{\alpha-1}}=\lambda E\left[I_{1}(T)\right]=\lambda Q(T / 2) .
$$

Controlling term $I_{3}(T)$ is done via exploiting a specific property of Gaussian processes, namely that $S_{s}$ for $s>t$ can be decomposed as $S_{s}=\rho(s, t) S_{t}+W_{s, t}$, where $W_{s, t}$ is independent of $S_{t}$ and zero mean. With this, observe that

$$
\begin{aligned}
E I_{3}(T)= & \frac{1}{T / 2} \sum_{s=T / 2+1}^{T} \sum_{t=0}^{T / 2} E\left[\rho(s, t) S_{t} \operatorname{sgn}\left(S_{t}\right)\left|S_{t}\right|^{\frac{1}{\alpha-1}}\right] \\
& =\frac{1}{T / 2} \sum_{s=T / 2+1}^{T} \sum_{t=0}^{T / 2} E\left[\rho(s, t)\left|S_{t}\right|^{\frac{\alpha}{\alpha-1}}\right] .
\end{aligned}
$$

Let the constants $\bar{T}, R, K, \eta=2 / 3$ and $\varepsilon$ be as in Lemma 4.4, and decompose the double sum in (4.16) as

$$
\sum_{s=T / 2+1}^{T} \sum_{t=0}^{T / 2}=\sum_{s=T / 2+1}^{T} \sum_{t=0}^{\bar{T}-1}+\sum_{s=T / 2+1}^{T / 2+K} \sum_{t=\bar{T}}^{T / 2}+\sum_{s=T / 2+K+1}^{\eta T} \sum_{t=\bar{T}}^{T / 2}+\sum_{s=\eta T+1}^{T} \sum_{t=\bar{T}}^{T / 2}
$$


Note that applying the upper bound developed in Lemma 4.4 to the double sum in (4.16), the summand no longer depends on the running variable of the outer sum. Denoting $C_{\bar{T}}:=\sum_{t=0}^{\bar{T}-1} E\left|S_{t}\right|^{\frac{\alpha}{\alpha-1}}$, this implies that

$$
\begin{aligned}
E I_{3}(T) & \leq\left(\sum_{t=0}^{T / 2}+R \sum_{t=0}^{\bar{T}-1}+\frac{2 R K}{T} \sum_{t=\bar{T}}^{T / 2}-2 \varepsilon\left(1-\frac{2}{3}\right) \sum_{t=\bar{T}}^{T / 2}\right) E\left|S_{t}\right|^{\frac{\alpha}{\alpha-1}} \\
& =E\left[I_{1}(T)\right]+\left(R \sum_{t=0}^{\bar{T}-1}+\frac{2 R K}{T} \sum_{t=\bar{T}}^{T / 2}-\frac{2 \varepsilon}{3} \sum_{t=\bar{T}}^{T / 2}\right) E\left|S_{t}\right|^{\frac{\alpha}{\alpha-1}} \\
& =E\left[I_{1}(T)\right]+\left(\left(R+\frac{2 \varepsilon}{3}-\frac{2 R K}{T}\right) \sum_{t=0}^{\bar{T}-1}+\frac{2 R K}{T} \sum_{t=0}^{T / 2}-\frac{2 \varepsilon}{3} \sum_{t=0}^{T / 2}\right) E\left|S_{t}\right|^{\frac{\alpha}{\alpha-1}} \\
& =\left(1-\frac{2 \varepsilon}{3}\right) E\left[I_{1}(T)\right]+\left(R+\frac{2 \varepsilon}{3}-\frac{2 R K}{T}\right) C_{\bar{T}}+\frac{2 R K}{T} E\left[I_{1}(T)\right],
\end{aligned}
$$

so we have

$$
E\left[I_{1}(T)\right]-E\left[I_{3}(T)\right] \geq \frac{2 \varepsilon}{3} E\left[I_{1}(T)\right]-\left(R+\frac{2 \varepsilon}{3}-\frac{2 R K}{T}\right) C_{\bar{T}}-\frac{2 R K}{T} E\left[I_{1}(T)\right]
$$

The above, using (4.15), boils down to

$$
X_{T}(\phi) \geq \frac{2 \varepsilon}{3} Q(T / 2)-\left(R+\frac{2 \varepsilon}{3}-\frac{2 R K}{T}\right) C_{\bar{T}}-\frac{2 R K}{T} Q(T / 2)-2 \lambda Q(T / 2)
$$

Using (4.13) and (4.14), with $\lambda<\varepsilon / 3$, dividing through with $T^{H\left(1+\frac{1}{\alpha-1}\right)+1}$ proves the statement in (3.5) with the constraint that the limiting operation runs through multiples of 6 . Now let $T$ be general. The same calculations can be done as above, with minor changes in the formulas corresponding to the upper and lower limits in summations according to taking the appropriate floor values. That is, in the last inequality $Q(3\lfloor T / 6\rfloor)$ appears - instead of $Q(T / 2)$ - and it grows in the order of $(6\lfloor T / 6\rfloor)^{H\left(1+\frac{1}{\alpha-1}\right)+1}$, and using that $6\lfloor T / 6\rfloor / T$ tends to 1 when $T$ is large, the proof of Theorem 3.3, noting $\chi=2 H-2$, is complete.

\section{References}

[1] R. Almgren and N. Chriss. Optimal execution of portfolio transactions. Journal of Risk, 3:5-40, 2001.

[2] R. T. Baillie. Long memory processes and fractional integration in econometrics. J. Econometrics, 73:5-59, 1996. MR-1410000

[3] D. Bertsimas and A. Lo. Optimal control of execution costs. Journal of Financial Markets, 1:1-50, 1998.

[4] F. Comte and E. Renault. Long memory in continuous-time stochastic volatility models. Math. Finance, 8:291-323, 1998. MR-1645101

[5] Y. Dolinsky and H. M. Soner. Duality and Convergence for Binomial Markets with Friction. Finance Stoch., 17:447-475, 2013. MR-3066984

[6] N. Garleanu and L. Pedersen. Dynamic trading with predictable returns and transaction costs. Journal of Finance, 68:2309-2340, 2013.

[7] J. Gatheral, T. Jaisson and M. Rosenbaum. Volatility is rough. Quantitative Finance, 18:933949, 2018. MR-3805308

[8] L. Giraitis, H. L. Koul and D. Surgailis. Large Sample Inference for Long Memory Processes. Imperial College Press, 2012. MR-2977317 
Long-term investment for prices with negative memory

[9] P. Guasoni. No arbitrage under transaction costs, with fractional Brownian motion and beyond. Mathematical Finance, 16:569-582, 2006. MR-2239592

[10] P. Guasoni, Zs. Nika and M. Rásonyi. Trading fractional Brownian motion. SIAM J. Financial Mathematics, 10:769-789, 2019. MR-4000210

[11] P. Guasoni and M. Rásonyi. Hedging, arbitrage and optimality under superlinear friction. Annals of Applied Probability, 25:2066-2095, 2015. MR-3349002

[12] O. Hernandez-Lerma and J.-B. Lasserre. Discrete-Time Markov Control Processes. Springer, 1996. MR-1363487

[13] A. S. Kyle. Continuous auctions and insider trading. Econometrica, 29:1315-1335, 1985.

[14] T. Leung and B. Ward. Tracking VIX with VIX Futures: Portfolio Construction and Performance. Preprint, 2019. arXiv:1907.00293

[15] B. B. Mandelbrot. When can price be arbitraged efficiently? A limit to the validity of the random walk and martingale models. The Review of Economics and Statistics, 53:225-236, 1971. MR-0378737

[16] L. C. G. Rogers. Arbitrage with fractional Brownian motion. Mathematical Finance, 7:95-105. 1997. MR-1434408

[17] L. C. G. Rogers and S. Singh. The cost of illiquidity and its effects on hedging. Math. Finance, 20:597-615, 2010. MR-2731409

[18] W. Schachermayer and J. Teichmann. How close are the Option Pricing Formulas of Bachelier and Black-Merton-Scholes? Mathematical Finance, 18:155-170, 2008. MR-2380944 